\title{
Use of Rc4 Method In Android-Based Sms Security Application
}

\author{
Oktovantua Butarbutar \\ Teknik Informatika, STMIK Jakarta STI\&K \\ Email: oktovantua45@gmail.com
}

\begin{tabular}{ll}
\hline Keywords & $\begin{array}{l}\text { Abstract. In maintaining the confidentiality of SMS, it takes a way to secure information } \\
\text { that is important or confidential, namely by encrypting the SMS text, the level of } \\
\text { information security of the message can be increased. One way to use for data and or } \\
\text { information security is to use cryptographic system, this application uses RC4 algorithm } \\
\text { which has the advantage of having a high level of security and speed process. This } \\
\text { makes RC4 the best choice for the encryption process required by the information world } \\
\text { towards the next century. The purpose ofbya is to prevent the occurrence of information / } \\
\text { data taker without being known by the owner. }\end{array}$ \\
\hline
\end{tabular}

\section{INTRODUCTION}

Data security and confidentiality issues are one of the most important aspects of an information system. This is very much related to how important the information is to be sent and received by people interested in the data. Information will no longer be useful if in the middle of the way data is hijacked or intercepted by unauthorized persons [1]. The information on the data will be lost so that the recipient will get different information. One of the ways used for data or information security is to use cryptographic systems, this application uses RC4 algorithms that have the advantage of having a high level of security and speed processes [2]. This makes RC4 the best choice for the encryption process required by the information world towards the next step. In maintaining the confidentiality of SMS, it takes a way to secure information that is important or confidential, namely by encrypting the SMS text, the level of information security of the message can be increased. The author in addressing the problem of sending messages, trying to create a messaging application with RC4 algorithm to encrypt data running on android operating system so that owners of android-based mobile phones (phones) can exchange SMS data more safely and conveniently [1], [3].

A text messaging service component of most telephones, Internet, and mobile-device systems is known as short message service (SMS) [4]. Standardized communication protocols are used to permit smart phones to transfer short text messages. Short message service is also commonly referred to as a "text message." The user can conduct a message of up to 160 characters to another device with a SMS. In SMS, longer messages will automatically be fragmented into several parts. This type of text messaging is supported by most cell phones. The formal name for text messaging is SMS. Short message service is a way to conduct short, text-only messages from one phone to another. These messages are usually conducted over a cellular data network. The procedure for conducting SMS is launching the Messages application on the phone. Tap on the Compose Message button. Enter the phone number or name of the contact you want to text. Type your message and finally hit Send. These days, there exist a number of security issues and vulnerabilities related to SMS [3].

\section{METHOD}

\subsection{Rc4 Method}

Cryptography is derived from the Greek "Cryptos" meaning "Secret" and "graphein" means "writing". So, cryptography means "Secret Writing". Definition stated in [SCH96] : Cryptography is the science and art of keeping messages safe. The Rivest Code 4 (RC4) cryptographic algorithm is one of the symmetric key algorithms created by RSA Data Security Inc (RSADSI) in the form of a chpper stream [4]. The algorithm was invented in 1987 by Ronald Rivest and became a symbol of 
RSA security (short for three inventor names: Rivest Shamir Adleman) [10]. RC4 uses akey length from 1 to 256 bytesthat is used to initialize a 256-byte table. This table is used for the following generations of pseudo randoms that use XOR with plaintext to generate ciphertext [5], [6]. Each element in the table is exchanged at least once. RC4 is one type of ciper stream, which is processing units or data inputs at a time. In this way encryption or decryption can be implemented at variable lengths. This algorithm does not have to wait for a certain amount of data input before it is processed, or add additional bytes to encrypt. Rc4 encryption methods are very fast approximately 10 times faster than DES.

\subsection{RC4 Encryption and Decryption}

The encryption and decryption processes have the same process so that only one function is performed to run both processes. The following will be given a section describing the series of processes executed to describe or decrypt [8]:

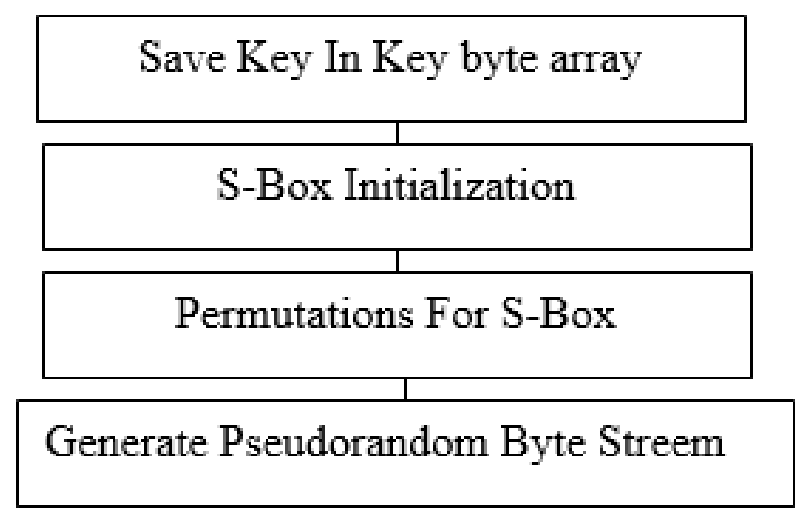

Figure 1 Encryption and Decryption Process

\section{RESULTS AND DISCUSSION}

\subsection{Encryption-Decryption Process Analysis}

Rc4 Stream Cipher algorithm to perform encryption, the process begins with the initialization of the first Sbox, $S$ [0], S [1],...., S [255], with the numbers 0 to 255 . First fill sequentially $[0]=0, S$ $[1]=1, \ldots ., S[255]=255$. Then initialize another array (another S-Box), e.g. array $\mathrm{K}$ with a length of 256. Fill array $\mathrm{K}$ with the keys to repeat until the entire array K [0], K [1],...., K [255] are fully populated.

1. S-Box initialization process (Array $\mathrm{S}$ )

$$
\begin{aligned}
& \text { For } \mathrm{i}=0 \text { to } 255 \\
& \mathrm{~S} \text { [i] }=\mathrm{i}
\end{aligned}
$$

2. S-Box initialization process (Array K)

// Array key array with key length "leght"

For $\mathrm{i}=0$ to 255

$$
\mathrm{S}[\mathrm{i}]=\mathrm{i}
$$

3. Then perform the S-Box randomization step

$$
\begin{aligned}
& \mathrm{I}=0 ; j=0 \\
& \text { For } \mathrm{i}=0 \text { to } 255 \\
& \begin{array}{l}
\{\quad \mathrm{J}=(\mathrm{j}+\mathrm{S}[\mathrm{i}]+[\mathrm{k}] \bmod 256) \\
\quad \text { Swap } S[\mathrm{i}] \text { And } S[\mathrm{j}]
\end{array} \\
& \quad
\end{aligned}
$$

4. Create a byte pseudorandom

$$
\begin{aligned}
& \mathrm{i}=(\mathrm{i}+1) \bmod 256 \\
& \mathrm{j}=(\mathrm{j}+\mathrm{S}[\mathrm{i}]) \bmod 256 \\
& \text { swap S [i] And } \mathrm{S} \text { [j] }
\end{aligned}
$$

Jurnal Info Sains : Informatikan dan Sains is licensed under a Creative Commons Attribution-Non Commercial 4.0 International License (CC BY-NC 4.0) 


$$
\begin{aligned}
& \mathrm{t}=(\mathrm{S}[\mathrm{i}]+\mathrm{S}[\mathrm{j}]) \bmod 256 \\
& \mathrm{~K}=\mathrm{S}[\mathrm{t}]
\end{aligned}
$$

5. Byte $\mathrm{K}$ is XOR-kan with plaintext to produce ciphertext or in-XOR-kan with ciphertext to produce plaintext.

Here is the implementation of RC4 algorithm with 256 bytes mode

1.Initialization of $S$-Box with a length of 256 bytes, with $S[0]=0, S[1]=1$, $\mathrm{S}[2]=2$ and $\mathrm{S}[3]=3, \ldots . ., \mathrm{S}[255]$ so that array $\mathrm{S}$ becomes:

Table 1. S-Box with a length of 256 bytes

\begin{tabular}{|l|l|l|l|l|l|l|l|l|l|l|l|l|l|l|l|}
\hline 0 & 1 & 2 & 3 & 4 & 5 & 6 & 7 & 8 & 9 & 10 & 11 & 12 & 13 & 14 & 15 \\
\hline 16 & 17 & 18 & 19 & 20 & 21 & 22 & 23 & 24 & 25 & 26 & 27 & 28 & 29 & 30 & 31 \\
\hline 32 & 33 & 34 & 35 & 36 & 37 & 38 & 39 & 40 & 41 & 42 & 43 & 44 & 45 & 46 & 47 \\
\hline 48 & 49 & 50 & 51 & 52 & 53 & 54 & 55 & 56 & 57 & 58 & 59 & 60 & 61 & 62 & 63 \\
\hline 64 & 65 & 66 & 67 & 68 & 69 & 70 & 71 & 72 & 73 & 74 & 75 & 76 & 77 & 78 & 79 \\
\hline 80 & 81 & 82 & 83 & 84 & 85 & 86 & 87 & 88 & 89 & 90 & 91 & 92 & 93 & 94 & 95 \\
\hline 96 & 97 & 98 & 99 & 100 & 101 & 102 & 103 & 104 & 105 & 106 & 107 & 108 & 109 & 110 & 111 \\
\hline 112 & 113 & 114 & 115 & 116 & 117 & 118 & 119 & 120 & 121 & 122 & 123 & 124 & 125 & 126 & 127 \\
\hline 128 & 129 & 130 & 131 & 132 & 133 & 134 & 135 & 136 & 137 & 138 & 139 & 140 & 141 & 142 & 143 \\
\hline 144 & 145 & 146 & 147 & 148 & 149 & 150 & 151 & 152 & 153 & 154 & 155 & 156 & 157 & 158 & 159 \\
\hline 160 & 161 & 162 & 163 & 164 & 165 & 166 & 167 & 168 & 169 & 170 & 171 & 172 & 173 & 174 & 175 \\
\hline 176 & 177 & 178 & 179 & 180 & 181 & 182 & 183 & 184 & 185 & 186 & 187 & 188 & 189 & 190 & 191 \\
\hline 192 & 193 & 194 & 195 & 196 & 197 & 198 & 199 & 200 & 201 & 202 & 203 & 204 & 205 & 206 & 207 \\
\hline 208 & 209 & 210 & 211 & 212 & 213 & 214 & 215 & 216 & 217 & 218 & 219 & 220 & 221 & 222 & 223 \\
\hline 224 & 225 & 226 & 227 & 228 & 229 & 230 & 231 & 232 & 233 & 234 & 235 & 236 & 237 & 238 & 239 \\
\hline 240 & 241 & 242 & 243 & 244 & 245 & 246 & 247 & 248 & 249 & 250 & 251 & 252 & 253 & 254 & 255 \\
\hline
\end{tabular}

2. Initialize the 10-byte key array, Ki. Suppose the key is taken from 10 bytes i.e. "OPEN[space]key" then the sentence will be converted into Decimal form "66 85756532107117 11099 105". Repeat the key until it meets the entire K array so that array K becomes:

$\begin{array}{llllllllll}\text { Iter-iKey-charKey[i]Sbox [i] } & & 23 & \text { K } & 75 & 22 & \\ 1 & \text { B } & 66 & 0 & 24 & \text { A } & 65 & 23 & \\ 2 & \text { U } & 85 & 1 & 25 & \text { [space] } & 32 & 24 \\ 3 & \text { K } & 75 & 2 & 26 & \text { K } & 107 & 25 & \\ 4 & \text { A } & 65 & 3 & & 27 & \text { U } & 117 & 26 & \\ 5 & \text { [space] } & 32 & 4 & 28 & \text { N } & 110 & 27 & \\ 6 & \text { K } & 107 & 5 & & 29 & \text { C } & 99 & 28 & \\ 7 & \text { U } & 117 & 6 & 30 & \text { I } & 105 & 29 & \\ 8 & \text { N } & 110 & 7 & & 31 & \text { B } & 66 & 30 & \\ 9 & \text { C } & 99 & 8 & & 32 & \text { U } & 85 & 31 & \\ 10 & \text { I } & 105 & 9 & & 33 & \text { K } & 75 & 32 & \\ 11 & \text { B } & 66 & 10 & & 34 & \text { A } & 65 & 33 & \\ 12 & \text { U } & 85 & 11 & & 35 & \text { [space] } & 32 & 34 \\ 13 & \text { K } & 75 & 12 & & 36 & \text { K } & 107 & 35 & \\ 14 & \text { A } & 65 & 13 & 37 & \text { U } & 117 & 36 & \\ 15 & \text { [space] } & 32 & 14 & 38 & \text { N } & 110 & 37 & \\ 16 & \text { K } & 107 & 15 & & 39 & \text { C } & 99 & 38 & \\ 17 & \text { U } & 117 & 16 & 40 & \text { I } & 105 & 39 & \\ 18 & \text { N } & 110 & 17 & 41 & \text { B } & 66 & 40 & \\ 19 & \text { C } & 99 & 18 & 42 & \text { U } & 85 & 41 & \\ 20 & \text { I } & 105 & 19 & 43 & \text { K } & 75 & 42 & \\ 21 & \text { B } & 66 & 20 & 44 & \text { A } & 65 & 43 & \\ 22 & \text { U } & 85 & 21 & 45 & \text { [space] } & 32 & 44\end{array}$

Jurnal Info Sains : Informatikan dan Sains is licensed under a Creative Commons Attribution-Non Commercial 4.0 International License (CC BY-NC 4.0) 


\begin{tabular}{|c|c|c|}
\hline 46 & K 107 & 45 \\
\hline 47 & U 117 & 46 \\
\hline 48 & N 110 & 47 \\
\hline 49 & C 99 & 48 \\
\hline 50 & I 105 & 49 \\
\hline 51 & В 66 & 50 \\
\hline 52 & U 85 & 51 \\
\hline 53 & K 75 & 52 \\
\hline 54 & A 65 & 53 \\
\hline 55 & [space] & 32 \\
\hline$\cdots \cdot$ & $\cdots \cdot \cdots$ & $\cdots$ \\
\hline$\cdots \cdot$ & $\cdots \cdot \cdots$ & $\cdots \cdot$ \\
\hline .... & $\cdots \cdot \cdots$ & $\cdots \cdot$ \\
\hline 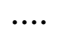 & $\ldots . \cdots$ & $\ldots$ \\
\hline-1 & [space] & 32 \\
\hline 256 & K 107 & 255 \\
\hline
\end{tabular}

3. Next mix the operation where it will use the variable $i$ And $j$ to index array $S$ [i] And $K$ [i]. First we give the initial value for $\mathrm{i}$ And $\mathrm{j}$ with 0 . The mixing operation is a repeat of the formula ( $\mathrm{i}+\mathrm{S}[\mathrm{i}]+\mathrm{K}[\mathrm{j}]$ ) $\bmod 256$ followed by the exchange of $S[i]$ with $S$ [j]. For this example, because we are using an array with a length of 256 bytes so the algorithm becomes:

$$
\text { For } \begin{aligned}
i & =0 \text { to } 256 \\
j & =(j+S[i]+K[i]) \bmod 256
\end{aligned}
$$

swap $\mathrm{S}$ [i] And $\mathrm{S}$ [j]

4. With the algorithm as above So with the initial value $i=0$ to $i=256$ will generate an array of $\mathrm{S}$ as below:

Iteration to1:

$$
\begin{aligned}
\mathrm{i} & =0, \text { So } \\
\mathrm{j} & =(\mathrm{j}+\mathrm{S}[\mathrm{i}]+\mathrm{K}[\mathrm{i}] \bmod 256 \\
& =(\mathrm{j}+\mathrm{S}[0]+\mathrm{K}[0] \bmod 256 \\
& (0+0+66) \bmod 256 \\
& =66
\end{aligned}
$$

Swap S [0] And S [66]

Iteration to2:

$$
\begin{aligned}
\mathrm{i}= & 0, \text { So } \\
\mathrm{j}= & (\mathrm{j}+\mathrm{S}[\mathrm{i}]+\mathrm{K}[\mathrm{i}] \bmod 256 \\
= & (\mathrm{j}+\mathrm{S}[1]+\mathrm{K}[1] \bmod 256 \\
& (66+1+85) \bmod 256 \\
= & 152
\end{aligned}
$$

Swap S [1] And S [152]

Iteration to3:

$$
\begin{aligned}
\mathrm{i} & =1, \text { So } \\
\mathrm{j} & =(\mathrm{j}+\mathrm{S}[\mathrm{i}]+\mathrm{K}[\mathrm{i}] \bmod 256 \\
& =(\mathrm{j}+\mathrm{S}[2]+\mathrm{K}[2] \bmod 256 \\
& (152+2+75) \bmod 256 \\
& =229
\end{aligned}
$$

Swap S [2] And S [229]
Iteration to4:

$$
\begin{aligned}
\mathrm{i} & =3, \text { So } \\
\mathrm{j} & =(\mathrm{j}+\mathrm{S}[\mathrm{i}]+\mathrm{K}[\mathrm{i}] \bmod 256 \\
& =(\mathrm{j}+\mathrm{S}[3]+\mathrm{K}[3] \bmod 256 \\
& (229+3+65) \bmod 256 \\
& =41
\end{aligned}
$$

Swap S [3] And S [41]

Iteration to5:

$$
\begin{aligned}
\mathrm{i} & =4, \text { So } \\
\mathrm{j} & =(\mathrm{j}+\mathrm{S}[\mathrm{i}]+\mathrm{K}[\mathrm{i}] \bmod 256 \\
& =(\mathrm{j}+\mathrm{S}[4]+\mathrm{K}[4] \bmod 256 \\
& =(41+4+32) \bmod 256 \\
& =77
\end{aligned}
$$

Swap S [4] And S [77]

Iteration to6:

$$
\begin{aligned}
\mathrm{i} & =5, \text { So } \\
\mathrm{j} & =(\mathrm{j}+\mathrm{S}[\mathrm{i}]+\mathrm{K}[\mathrm{i}] \bmod 256 \\
& =(\mathrm{j}+\mathrm{S}[5]+\mathrm{K}[5] \bmod 256 \\
& =(77+5+107) \bmod 256 \\
& =189
\end{aligned}
$$

Swap S [5] And S [189]

Iteration to 7 :

$$
\begin{aligned}
\mathrm{i} & =6, \text { So } \\
\mathrm{j} & =(\mathrm{j}+\mathrm{S}[\mathrm{i}]+\mathrm{K}[\mathrm{i}] \bmod 256 \\
& =(\mathrm{j}+\mathrm{S}[6]+\mathrm{K}[6] \bmod 256 \\
& =(189+6+117) \bmod 256 \\
& =56
\end{aligned}
$$

Swap S [6] And S [56]

Iteration to 8:

$$
\begin{aligned}
\mathrm{i} & =7, \text { So } \\
\mathrm{j} & =(\mathrm{j}+\mathrm{S}[\mathrm{i}]+\mathrm{K}[\mathrm{i}] \bmod 256 \\
& =(\mathrm{j}+\mathrm{S}[7]+\mathrm{K}[7] \bmod 256 \\
& =(56+7+110) \bmod 256 \\
& =173
\end{aligned}
$$

Swap S [7] And S [173]

Iteration to9:

$$
\begin{aligned}
\mathrm{i} & =8, \mathrm{So} \\
\mathrm{j} & =(\mathrm{j}+\mathrm{S}[\mathrm{i}]+\mathrm{K}[\mathrm{i}] \bmod 256 \\
& =(\mathrm{j}+\mathrm{S}[8]+\mathrm{K}[8] \bmod 256 \\
& =(173+8+99) \bmod 256 \\
& =24
\end{aligned}
$$

Swap S [8] And S [24]

Iteration to10:

$$
\begin{aligned}
\mathrm{i} & =9, \text { So } \\
\mathrm{j} & =(\mathrm{j}+\mathrm{S}[\mathrm{i}]+\mathrm{K}[\mathrm{i}] \bmod 256 \\
& =(\mathrm{j}+\mathrm{S}[9]+\mathrm{K}[9] \bmod 256 \\
& =(24+9+105) \bmod 256 \\
& =138
\end{aligned}
$$

Swap S [9] And S [138]

Iteration to254:

$$
\begin{aligned}
& \mathrm{i}=253, \text { So } \\
& \mathrm{j}=(\mathrm{j}+\mathrm{S}[\mathrm{i}]+\mathrm{K}[\mathrm{i}] \bmod 256
\end{aligned}
$$

Jurnal Info Sains : Informatikan dan Sains is licensed under a Creative Commons Attribution-Non Commercial 4.0 International License (CC BY-NC 4.0) 


$$
\begin{aligned}
& =(j+S[253]+K[253] \bmod 256 \\
& =(27+67+65) \bmod 256 \\
& =159
\end{aligned}
$$

Swap S [5] And S [159]

Iteration to255:

$$
\begin{aligned}
\mathrm{i} & =254, \mathrm{So} \\
\mathrm{j} & =(\mathrm{j}+\mathrm{S}[\mathrm{i}]+\mathrm{K}[\mathrm{i}] \bmod 256 \\
& =(\mathrm{j}+\mathrm{S}[254]+\mathrm{K}[254] \bmod 256 \\
& =(159+99+32) \bmod 256
\end{aligned}
$$

$$
=34
$$

Swap S [5] And S [34]

Iteration to256:

$$
\begin{aligned}
\mathrm{i} & =255, \text { So } \\
\mathrm{j} & =(\mathrm{j}+\mathrm{S}[\mathrm{i}]+\mathrm{K}[\mathrm{i}] \bmod 256 \\
& =(\mathrm{j}+\mathrm{S}[255]+\mathrm{K}[255] \bmod 256 \\
& =(34+94+107) \bmod 256 \\
& =235
\end{aligned}
$$

Swap S [255] And S [235]

5. Next is the encryption process which is XOR-kan pseudorandom byte with plaintext, e.g. plaintext "ITU[space]DIA". Plaintext consists of 7 characters So occurs 7 iterations. Before iterating, convert the characters to binary number forms.

\begin{tabular}{lrllll}
\multicolumn{2}{l}{ Character } & Decimal & Biner \\
I & 73 & 0100 & 1001 & \\
T & 84 & 0101 & 0100 & \\
U & 85 & 0101 & 0101 & \\
[space] & 32 & 00100000 & \\
D & 68 & 0100 & 0100 & \\
I & 73 & 0100 & 1001 & \\
A & 65 & 0100 & 0001 &
\end{tabular}

Here's Iteration to1: Initialize $\mathrm{i}$ And $\mathrm{j}$ with $\mathrm{i}=0 ; \mathrm{j}$ $=0$;

$$
\begin{aligned}
\mathrm{i} & =(\mathrm{i}+1) \bmod 256 \\
& =(0+1) \\
& =1 \\
\mathrm{j} & =(\mathrm{j}+\mathrm{S}[\mathrm{i}] \bmod 256) \\
& =(\mathrm{j}+\mathrm{S}[1]) \bmod 256 \\
& =(0+44) \bmod 256 \\
& =44
\end{aligned}
$$$$
\text { And }
$$

Swap S[1] And S[44]

$$
\begin{aligned}
\mathrm{t} & =(\mathrm{S}[\mathrm{i}]+\mathrm{S}[\mathrm{j}]) \bmod 256 \\
& =(\mathrm{S}[1]+\mathrm{S}[44]) \bmod 256 \\
& =(71+44) \bmod 256
\end{aligned}
$$$$
=115
$$

$\mathrm{K}=\mathrm{S}[\mathrm{t}]=\mathrm{S}[15]=14=00001110$

Byte K di-XOR-with plaintext "I"

Plainteks I

73

01001001

Key ( K ) $\quad 00001110$

XOR 01000111

Chipertext $\quad 71$

$\mathrm{G}$

Iteration to2:

$$
\begin{aligned}
& \begin{aligned}
\mathrm{i} & =(\mathrm{i}+1) \bmod 256 \\
& =(1+1) \\
& =2
\end{aligned} \\
& \text { And } \\
& \quad \mathrm{j}
\end{aligned}
$$

$$
\begin{aligned}
& =(44+\mathrm{S}[2]) \bmod 256 \\
& =(44+229) \bmod 256 \\
& =17
\end{aligned}
$$

Swap S[2] And S[17]

$$
\begin{aligned}
\mathrm{t} & =(\mathrm{S}[\mathrm{i}]+\mathrm{S}[\mathrm{j}]) \bmod 256 \\
& =(\mathrm{S}[2]+\mathrm{S}[17]) \bmod 256 \\
& =(117+229) \bmod 256
\end{aligned}
$$$$
=90
$$

$\mathrm{K}=\mathrm{S}[\mathrm{t}]=\mathrm{S}[90]=202=10001000$

Byte K di-XOR with plaintext "T"

Plainteks T

84

01000100

Key ( K ) $\quad 00000010$

XOR 01011100

Chipertext 92

Iteration to3:

$$
\begin{aligned}
\mathrm{i} & =(\mathrm{i}+1) \bmod 256 \\
& =(2+1) \\
& =3
\end{aligned}
$$

And

$$
\begin{aligned}
\mathrm{j} & =(\mathrm{j}+\mathrm{S}[\mathrm{i}] \bmod 256) \\
& =(\mathrm{j}+\mathrm{S}[3]) \bmod 256 \\
& =(17+160) \bmod 256 \\
& =177
\end{aligned}
$$

Swap S[3] And S[177]

$$
\begin{aligned}
\mathrm{t} & =(\mathrm{S}[\mathrm{i}]+\mathrm{S}[\mathrm{j}]) \bmod 256 \\
& =(\mathrm{S}[3]+\mathrm{S}[15]) \bmod 256 \\
& =(12+160) \bmod 256
\end{aligned}
$$$$
=172
$$

$\mathrm{K}=\mathrm{S}[\mathrm{t}]=\mathrm{S}[172]=202=11001010$

Byte K di-XOR-with plaintext "U"

Plainteks U

85

01010101

Key ( K ) 11001010

XOR 10011111

Chipertext 159

Jurnal Info Sains : Informatikan dan Sains is licensed under a Creative Commons Attribution-Non Commercial 4.0 International License (CC BY-NC 4.0) 
Y

Iteration to4:

$$
\begin{aligned}
\mathrm{i} & =(\mathrm{i}+1) \bmod 256 \\
& =(3+1) \\
& =4 \\
\mathrm{j} & =(\mathrm{j}+\mathrm{S}[\mathrm{i}] \bmod 256) \\
& =(\mathrm{j}+\mathrm{S}[4]) \bmod 256 \\
& =(177+106) \bmod 256 \\
& =27
\end{aligned}
$$$$
\text { And }
$$

Swap S[4] And S[27]

$$
\begin{aligned}
\mathrm{t} & =(\mathrm{S}[\mathrm{i}]+\mathrm{S}[\mathrm{j}]) \bmod 256 \\
& =(\mathrm{S}[4]+\mathrm{S}[27]) \bmod 256 \\
& =(51+106) \bmod 256
\end{aligned}
$$$$
=157
$$

$\mathrm{K}=\mathrm{S}[\mathrm{t}]=\mathrm{S}[157]=75=01001010$

Byte K di-XOR-with plaintext "[Space]"

Plainteks [Space]

32

00100000

Key ( K ) 01001011

XOR 01101011

Chipertext 107

$\mathrm{K}$

$$
\begin{aligned}
& \text { Iteration to5: } \\
& \qquad \begin{aligned}
\mathrm{i} & =(\mathrm{i}+1) \bmod 256 \\
& =(4+1) \\
& =5 \\
\text { And } & \\
\mathrm{j} & =(\mathrm{j}+\mathrm{S}[\mathrm{i}] \bmod 256) \\
& =(\mathrm{j}+\mathrm{S}[5]) \bmod 256 \\
& =(27+185) \bmod 256 \\
& =212
\end{aligned}
\end{aligned}
$$

Swap S[5] And S[212]

$$
\begin{aligned}
\mathrm{t} & =(\mathrm{S}[\mathrm{i}]+\mathrm{S}[\mathrm{j}]) \bmod 256 \\
& =(\mathrm{S}[5]+\mathrm{S}[212]) \bmod 256 \\
& =(116+185) \bmod 256 \\
= & 45
\end{aligned}
$$

$\mathrm{K}=\mathrm{S}[\mathrm{t}]=\mathrm{S}[45]=223=11011111$

Byte K di-XOR-with plaintext " $D$ "

Plainteks

68

01000100

Key ( K )

XOR 10011011

Chipertext 155 1011111

$>$
Iteration to6:

$$
\begin{aligned}
\mathrm{i} & =(\mathrm{i}+1) \bmod 256 \\
& =(5+1) \\
& =6
\end{aligned}
$$

And

$\mathrm{j}=(\mathrm{j}+\mathrm{S}[\mathrm{i}] \bmod 256)$

$=(\mathrm{j}+\mathrm{S}[6]) \bmod 256$

$=(212+56) \bmod 256$

$=12$

Swap S[6] And S[12]

$$
\begin{aligned}
\mathrm{t} & =(\mathrm{S}[\mathrm{i}]+\mathrm{S}[\mathrm{j}]) \bmod 256 \\
& =(\mathrm{S}[6]+\mathrm{S}[12]) \bmod 256 \\
& =(141+56) \bmod 256
\end{aligned}
$$$$
=197
$$

$\mathrm{K}=\mathrm{S}[\mathrm{t}]=\mathrm{S}[197]=42=00101010$

Byte K di-XOR-with plaintext "I"

Plainteks I

73

01001001

Key ( K ) 00101010

XOR 01100011

Chipertext 99

$\mathrm{C}$

Iteration to $7:$

$$
\begin{aligned}
\mathrm{i} & =(\mathrm{i}+1) \bmod 256 \\
& =(6+1) \\
& =7 \\
\mathrm{j} & =(\mathrm{j}+\mathrm{S}[\mathrm{i}] \bmod 256) \\
& =(\mathrm{j}+\mathrm{S}[7]) \bmod 256 \\
& =(12+48) \bmod 256 \\
& =60
\end{aligned}
$$

And

Swap S[7] And S[60]

$$
\begin{aligned}
\mathrm{t} & =(\mathrm{S}[\mathrm{i}]+\mathrm{S}[\mathrm{j}]) \bmod 256 \\
& =(\mathrm{S}[7]+\mathrm{S}[60]) \bmod 256 \\
& =(64+48) \bmod 256
\end{aligned}
$$$$
=112
$$

$\mathrm{K}=\mathrm{S}[\mathrm{t}]=\mathrm{S}[112]=42=00100111$

Byte K di-XOR-with plaintext "A"

Plainteks A

65

01000001

Key ( K ) $\quad 00100111$

XOR 01100110

Chipertext 102

$\mathrm{F}$

So the results of encryption obtained after going through several iterations are as follows: len_tektiter-

Jurnal Info Sains : Informatikan dan Sains is licensed under a Creative Commons Attribution-Non Commercial 
iiter-

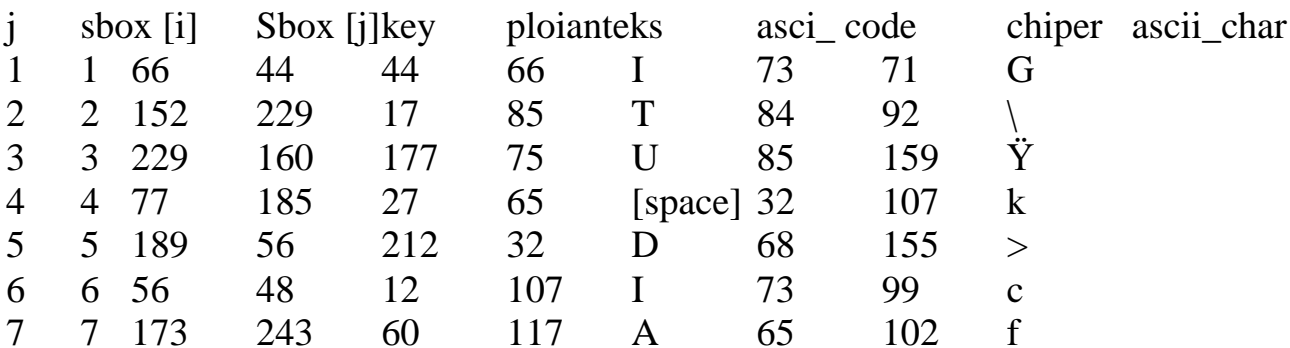

6. Next is the decryption process which is XOR-kan pseudo random byte with Chipertext, And Chipertext is "G $\backslash \ddot{\mathrm{Y}} \mathrm{k}>\mathrm{c} \mathrm{f}$ ". Chipertext consists of 7 characters so occurs 7 iterations. Before iterating, convert characters to binary number form

CharacterDecimalBiner

$\begin{array}{lll}\text { G } & 71 & 01000111 \\ \backslash & 92 & 01011100 \\ \ddot{Y} & 159 & 10011111 \\ \mathrm{k} & 107 & 01101011 \\ > & 155 & 10011011 \\ \mathrm{c} & 99 & 01100011 \\ \mathrm{f} & 102 & 01100110\end{array}$

The data is sent in the form of a chipertext so that once it reaches the recipient the message can be re-converted to plaintext by XOR-kan with the same key.

\section{Chipertext $\quad \mathrm{G} \backslash \ddot{\mathrm{Y}} \mathrm{k}>\mathrm{c} \mathrm{f}$ \\ Kunci BUKA [space] key \\ PlaintextITU [space] DIA}

Berikut iterasi 1:

Plaintext G

71

01000111

Key ( K ) $\quad 00001110$

XOR 01001001

Chipertext 73

I

Here's iteration 2 :

Plaintext ।

92

01011100

Key ( K ) $\quad 00001000$

XOR 01010100

Chipertext 84

$\mathrm{T}$

Berikut iterasi 3:

Plaintext $\quad \ddot{Y}$
159

10011111

Key ( K ) 11001010

XOR 01010101

Chipertext 85

$\mathrm{U}$

Here's iteration 4:

Plaintext K
107
01101011

Key ( K ) $\quad 01001011$

XOR 00100000

Chipertext 32

[Space]

Here's iteration 5:

Plaintext >
155
10011011

Key ( K ) $\quad 11011111$

XOR 01000100

Chipertext 68

D

Here's iteration 6:

Jurnal Info Sains : Informatikan dan Sains is licensed under a Creative Commons Attribution-Non Commercial 4.0 International License (CC BY-NC 4.0) 


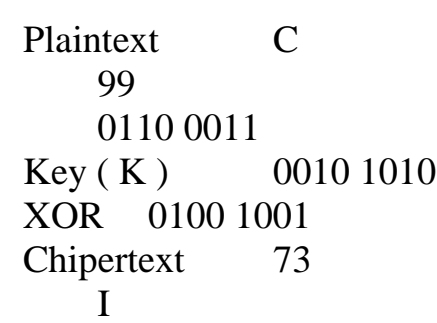

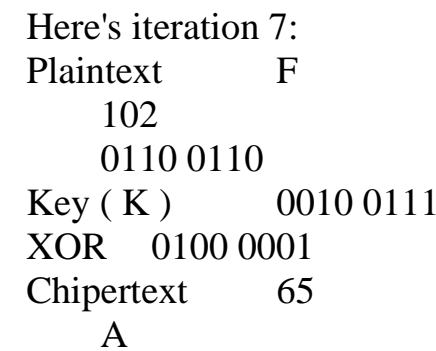

\section{CONCLUSION}

With aAndyes SMSencryption and decryption application with RC4 algorithm, it can provide convenience for users to encrypt SMS messages without having to do calculations. This application uses RC4 algorithm which has the advantage of having a high level of security and speed processes. Android-based SMS encryption apps can help users describe SMS messages before they're sent.

\section{REFERENCE}

[1] S. K. Fatima, S. G. Fatima, S. A. Sattar, and A. Sheela, "An advanced data security method in WSN," Int. J. Adv. Res. Eng. Technol., vol. 10, no. 2, 2019, doi: 10.34218/IJARET.10.2.2019.026.

[2] S. Pirbhulal, O. W. Samuel, W. Wu, A. K. Sangaiah, and G. Li, "A joint resourceaware and medical data security framework for wearable healthcare systems," Futur. Gener. Comput. Syst., vol. 95, 2019, doi: 10.1016/j.future.2019.01.008.

[3] R. Perangin-angin, I. K. Jaya, and ..., "Analisa Alokasi Memori dan Kecepatan Kriptograpi Simetris Dalam Enkripsi dan Dekripsi," J. Inf. ..., 2019.

[4] A. I. Sallam, O. S. Faragallah, and E. S. M. El-Rabaie, "HEVC Selective Encryption Using RC6 Block Cipher Technique," IEEE Trans. Multimed., vol. 20, no. 7, 2018, doi: 10.1109/TMM.2017.2777470.

[5] A. I. Sallam, E. S. M. El-Rabaie, and O. S. Faragallah, "CABAC-based selective encryption for HEVC using RC6 in different operation modes," Multimed. Tools Appl., vol. 77, no. 21, 2018, doi: 10.1007/s11042-018-5994-5.

[6] A. Subandi, M. S. Lydia, R. W. Sembiring, M. Zarlis, and S. Efendi, "Vigenere cipher algorithm modification by adopting RC6 key expansion and double encryption process," in IOP Conference Series: Materials Science and Engineering, 2018, vol. 420, no. 1, doi: 10.1088/1757-899X/420/1/012119.

[7] A. Ouertani et al., "Two new secreted proteases generate a casein-derived antimicrobial peptide in Bacillus cereus food born isolate leading to bacterial competition in milk," Front. Microbiol., vol. 9, no. JUN, 2018, doi: 10.3389/fmicb.2018.01148.

[8] Saranya et al., "Spritz - a spongy RC4-like stream cipher and hash function," IOP Conf. Ser. Mater. Sci. Eng., vol. 3, no. 3, 2018.

[9] Rusmala and D. Prasti, "Implementasi Metode Rail Fence Chiper dan Row Transposition Chiper Pada Mata Kuliah Kriptograpi," Ilm. d'Computare, vol. 9, 2019.

[10] A. Hidayat, K. A. Azhari, and D. Setiana, "Perbandingan Penggunaan Memory Dan Cpu Menggunakan Kriptograpi AES," JUTEKIN (Jurnal Tek. Inform., vol. 6, no. 2, 2018. 